\title{
Kampf um Rationalisierung - Suche nach neuer Übersichtlichkeit
}

Die Gewerkschaften setzen die Re-Vitalisierung der Arbeitspolitik auf ihre Agenda. Um welche neuen Schwerpunkte kann es dabei gehen? Wo sollte Schutzpolitik betrieben werden, die zumindest den Status quo sichert? Wo könnten eigene arbeitspolitische Konzepte offensiv in die Unternehmen eingebracht werden? Eine zentrale These des Artikels ist, dass die drei Arenen betrieblicher Rationalisierung, d.h. die Beschäftigungs-, Leistungs- und Arbeitspolitik, sehr unterschiedlichen Voraussetzungen und Gestaltungslogiken unterliegen. Für die Re-Vitalisierung der Gewerkschaftspolitik in diesen Feldern ist es wichtig, ihre Schwerpunktsetzung entlang der Spezifik der jeweiligen Gestaltungsoptionen zu entwickeln.

\section{Die Aufgabe: Re-Vitalisierung der Arbeitspolitik}

„Umkämpfte Arbeit“ titelt die Prokla ihre März-Ausgabe 2008. Die These lautet: „Arbeit ist in Deutschland wieder ins Zentrum gesellschaftlicher und politischer Auseinandersetzungen gerückt" (Prokla Redaktion 2008, S. 2). In einem Aufsatz des Hefts wird von Kratzer et al. diese Prämisse einer neuen Aktualität von Arbeit bestätigt. Während das letzte Jahrzehnt als eine Phase der „De-Thematisierung von Arbeit“ (Dunkel/Sauer 2006) und entsprechend als arbeitspolitisch verlorene Zeit einzuschätzen sei, habe sich nun die Lage gewandelt: „In jüngster Zeit geraten leistungspolitische Fragen durch steigenden Leistungsdruck in den Betrieben ... wieder mehr in den Fokus des öffentlichen Interesses" (Kratzer et al. 2008, S. 21).

Diese Einschätzung entspricht der Debattenlage in den Gewerkschaften. Auch bei der IG Metall wird etwa von einem "grundlegenden Wandel, der sich in den letzten Jahren in der Arbeitswelt vollzogen hat" (Huber/Schwitzer 2008, S. 7) ausgegangen. So beschloss die IG Metall auf ihrem letzten Gewerkschaftstag im November 2007, „Gute Arbeit“ zu einem Politikschwerpunkt der nächsten Jahre auszubauen (Fergen/Pickshaus 2008, S. 189ff.).

Wie diese Absichtserklärung in erfolgversprechende Politik umgesetzt werden kann, ist noch offen. In einem Thesenpapier für eine vorstandsinterne Generaldebatte wurden Unsicherheiten in der Lageeinschätzung von Berthold Huber ausdrücklich angesprochen. „Der weitere Ver- lauf der Globalisierung und seine Folgen sind schwer vorauszusagen. Jürgen Habermas beschreibt die Situation als ,neue Unübersichtlichkeit. ... In solchen ungeklärten Situationen haben Kämpfe um Deutungshoheit besonderes Gewicht" (Huber 2008, S. 4). Als Frage gelte es zu beantworten: „Wie können wir unter diesen Bedingungen stärker an einer Re-Vitalisierung der Arbeitspolitik arbeiten?“ (ebd. S. 7).

Mein Text stellt sich dieser Frage. Es geht mir um eine angemessene Beurteilung der aktuellen Rationalisierungslage in der deutschen Industrie. Meine These ist, dass sich hinter den nach wie vor geltenden Ungleichzeitigkeiten und Unbestimmtheiten erklärende Muster abzeichnen. Die Unübersichtlichkeit reduziert sich, wenn die verschiedenen, in Rationalisierung involvierten Politikebenen in ihren je eigenen Entwicklungsbedingungen betrachtet werden.

Rationalisierungspolitik geht es immer um Effizienzsteigerung. So wichtig dabei auch Technikentwicklungen sind: gerade unter dem Aspekt gewerkschaftlicher Gestaltungsmöglichkeiten haben daneben Beschäftigungs-, Leistungs- und Arbeitspolitik zentrale Bedeutung. Dabei gilt, dass diese drei Politikansätze zwar gleichermaßen demselben ökonomischen Ziel dienen, sie aber unterschiedlichen Voraussetzungen und Gestaltungslogiken unterliegen, was für ihre Konzeptionierung und Arbeitswirkung deutliche Differenzen begründet. Entsprechend müssen die Ansatzpunkte für eine Re-Vitalisierung der gewerkschaftlichen „Rationalisierungspolitik“ für die Beschäftigungs-, Leistungs- und Arbeitspolitik trotz aller inhaltlichen Wechselwirkungen und Verzahnungen auch getrennt konzipiert werden.

Jürgen Habermas hat in seinem viel zitierten Aufsatz über „Die Neue Unüber- sichtlichkeit" mit Verweis auf Gramsci's Konzept der kulturellen Hegemonie hervorgehoben, dass es dabei vor allem um den Kampf um Definitionen geht (Habermas 1985, S. 159). Der Hinweis auf „Kampf um Deutungshoheit" schließt hier an. Um für die Auseinandersetzung um Rationalisierung heute ein wirkungsvolles und durchsetzungsfähiges Konzept entwickeln zu können, sind die Reflexion bisheriger Erfahrungen wie auch begriffliche Vorklärungen nötig.

\section{Blick zurück}

Erinnern wir uns. Schon in den Rationalisierungsanalysen der vergangenen Jahrzehnte ging es - in den 1980er Jahren - um ein „Umbruchs-Szenarium“ und Ende der 1990er Jahre wurde aus arbeitspolitischer Perspektive von einer „Neuen Unübersichtlichkeit" gesprochen. Deswegen ist es sinnvoll, sich die damalige Debatte noch einmal kurz zu vergegenwärtigen.

\subsection{ARBEITSPOLITISCHER PARADIGMENWECHSEL IN DEN 1980ER JAHREN}

In Reaktion auf den in die Krise geratenen Taylorismus, für Jahrzehnte Leitbild kapi-

Michael Schumann, Prof. Dr., Präsident des Soziologischen Forschungsinstituts (SOFI) e. V. an der Universität Göttingen. Arbeitsschwerpunkte: Industrie- und Arbeitssoziologie, Industrielle Beziehungen e-mail: michael.schumann@sofi. uni-goettingen.de 
talistischer Rationalisierung und wichtigste Ursache für das sich ständig verbreitende Arbeitsleid, entwickelten die Unternehmen Ende der 1970er Jahre „Neue Produktionskonzepte". Wir sprachen am SOFI in Göttingen von einem arbeitspolitischen Paradigmenwechsel, der sich aus einer betrieblichen Neubewertung des menschlichen Faktors und einer grundlegend veränderten Nutzungsstrategie der Arbeitskraft ergab. In ihm drückte sich eine neue Wertschätzung der besonderen Qualitäten der menschlichen Arbeit aus. Produktionsintelligenz, unter tayloristischen Prämissen das zu eliminierende Ärgernis, bildete den zentralen Baustein dieser arbeitspolitischen Konzeption.

In der Arbeitsgestaltung meinte das vor allem Rücknahme von Arbeitsteilung, qualifikationsrelevante Funktionserweiterungen, Abbau von Kontrollmechanismen und mehr Selbststeuerung. In den Dimensionen der Leistungspolitik trat die neue Arbeitspolitik mit einem erweiterten betrieblichen Leistungsanspruch an. Wurden unter tayloristischen Prämissen Leistungssteigerungen vor allem durch Erhöhung des quantitativ fixierbaren Mengenoutputs pro Zeiteinheit angestrebt auf der Basis formalisierter, berechenbarer Fähigkeiten, so gehen in die neuen Produktionskonzepte nun auch nicht mehr rechnerisch festlegbare, qualitative Leistungskriterien ein wie insbesondere flexibles Arbeitsverhalten, selbstverantwortete Leistungssteuerung und Kooperationsbereitschaft (Dröge et al. 2008).

Für die arbeitspolitischen Veränderungen in den Unternehmen war die Erkenntnis bestimmend, dass man den Markterfordernissen und den Imperativen einer effizienten Nutzung der neuen Technologien am besten mit qualifizierten Arbeitskräften gerecht werde, eingesetzt in einer intelligenten, dynamischen Arbeitsorganisation. Der Rheinische Kapitalismus mit seinen hohen Entwicklungspotenzialen gerade für den Faktor Humanressourcen bot eine passgenaue institutionelle Plattform für die Entwicklung der neuen Produktionskonzepte; sie versprachen komparative Vorteile (Kern/Schumann 1984).

Da positive ökonomische Effekte mit dem Arbeitsvermögen und der Kreativität der Beschäftigten erzielt wurden - und nicht, wie früher oft, gegen diese -, waren sie geeignet, einen Rationalisierungskompromiss zu fundieren, der beiden Seiten etwas brachte: den Unternehmen verbes- serte Chancen im Wettbewerb und den Beschäftigten qualifizierte Arbeit und ordentliche Löhne. Dies setzte neue Prämissen für eine gewerkschaftliche Arbeitspolitik, die insbesondere auf der Unternehmensebene zwischen Betriebsräten und Modernisierern im Management gemeinsam erarbeitete und betrieblich umgesetzte Konzepte ermöglichte. Leistungspolitisch ging es zwar unterm Strich um Effizienzerhöhungen, doch durch die in der Arbeitsgestaltung angelegten verbesserten Regulationskonditionen waren sie nicht mehr gleichzusetzen mit Belastungssteigerungen.

Uns blieben damals Durchsetzungsschwierigkeiten, Widersprüchlichkeiten und neu entstehende Schattenseiten der neuen Produktionskonzepte keineswegs verborgen. Dennoch hatten wir gute Gründe, von einem arbeitspolitischen „Umbruch“ zu reden. In Aussicht stand für uns eine längere Inkubationsphase, doch letztlich erwarteten wir, dass sich der „neue Geist“ der Konzepte verbreitern und quasi in einer historischen Interessenallianz zwischen Kapital und Arbeit durchsetzen würde (ausführlich: Baethge et al. 1998).

Zusätzliches Gewicht bekam für die Gewerkschaften in diesen Jahren die Beschäftigungspolitik. Die ständig ansteigende Massenarbeitslosigkeit, die ins Zentrum der gesellschaftlich-politischen Auseinandersetzungen rückte, forderte sie nun in einer anderen Arena neu heraus.

\subsection{UNÜBERSICHTLICHKEIT IN DEN 1990ER JAHREN}

Dieser, wie wir damals meinten, feste Boden für eine einigermaßen verlässliche Aussage über die Zukunft der Arbeit hielt nicht allzu lange. Die politisch-ökonomischen Rahmenbedingungen veränderten sich unerwartet rasch. Beim Versuch einer ersten Bestandsaufnahme des zu beobachtenden Wandels titelte ich Ende der 1990er Jahre: „Rücknahme der Entwarnung - neue Gefährdungen der Industriearbeit" (Schumann 1998).

\section{AMBIVALENTE WIRKUNGEN NEUER PRODUKTIONSKONZEPTE}

Schon Ende der 1980er/Anfang der 1990er Jahre war durch unseren breitflächig angelegten "Trendreport Rationalisierung“ (Schumann et al. 1994) deutlich geworden, dass die neuen Produktionskonzepte mit ihrer Fortschrittsperspektive zwar für Teil- segmente der Beschäftigten Bedeutung gewannen, für große Mehrheiten aber der neue arbeitspolitische Ansatz nicht wirkte. Und Mitte der 1990er Jahre war in vielen Bereichen sogar eine Rekonventionalisierung zu beobachten. Ins Zentrum der arbeitspolitischen Gestaltung rückten erneut altbekannte Rezepte wie Arbeitsteilung, Standardisierung, Hierarchisierung, Kontrollverschärfung und MitwirkungsExklusion. Entsprechend konnte sich jedenfalls in diesen restrukturierten Feldern die Leistungspolitik erneut auf das Einklagen erhöhter Mengenoutputs konzentrieren (Kurz 1999).

Die weitere Nutzung des menschlichen Arbeitsvermögens entlang der neuen Produktionskonzepte, in der Industriesoziologie auch als „Subjektivierung von Arbeit“ diskutiert (zusammenfassend Moldaschl/ Voß 2002), zeigte zudem gerade in leistungspolitischer Perspektive janusköpfige Wirkungen: Mit dem Mehr an Selbstbestimmung korrespondierte nicht selten gesteigerte Selbstausbeutung. Die angebotenen Freiheitsgrade wurden vielfach leistungspolitisch einseitig instrumentalisiert. Und mit der aufgewerteten Arbeit traten auch neue Gefahren auf. Die Konzentration auf innovative Aufgaben und der Einsatz in hochflexiblen Feldern mit besonderen Lern- und Kreativitätsanforderungen konnte überfordern. Die Aufgaben selbstständiger Problemlösung sind dann mit neuen Unsicherheiten und Versagensängsten verbunden.

Für die Beschäftigungspolitik stand der weitere Arbeitsplatzabbau nach wie vor im Zentrum der Auseinandersetzungen. Doch auch in dieser Politikarena kam es zu neuen Herausforderungen. Prekäre Beschäftigungsformen, wie vor allem befristete Verträge, Zeit- und Leiharbeit sowie Niedriglohnjobs, gewannen immer mehr an Bedeutung.

\section{VERÄNDERTE RAHMENBEDIN- GUNGEN/GLOBALISIERUNG}

Wichtigste Ursachen für diesen Umschwung waren die weltweit sich durchsetzende Globalisierung des Wirtschaftens (welche durch die Auflösung des Ost-West-Konflikts noch einmal besondere ökonomische Schubkraft und zusätzliche politische Optionen erhielt), die durch wachsende Macht der Finanzmärkte sich verändernden Verwertungsstrategien der Unternehmen und die mit beiden Entwicklungen zusammenhän- 
gende, weiter steigende Massenarbeitslosigkeit. Die gesellschaftlich-politischen Kräfteverhältnisse verschoben sich zugunsten des Kapitals. Ein rigoroser werdendes Concession Bargaining mit schlechteren Karten für die Arbeitnehmerseite drückte den Machtverlust deutlich aus. Insbesondere die immer durchsetzungsfähigeren Shareholder-Value-Interessen, die bei den Unternehmen als steigende, kurzfristig einzulösende Renditeerwartungen ankamen, traten in Widerspruch zu der gerade erst erprobten neuen Modernität.

\section{HIGH-ROAD-STRATEGIEN VERSUS LOW COST}

Das deutsche Produktionsmodell, ein auf High-Road-Rationalisierung gründender post-fordistischer Klassenkompromiss, der seine besondere Stütze in der Verbindung von Produktivitäts- und Innovationspotenzialen mit hoch entwickelten Humanressourcen hatte, wurde von einigen Managern, mehr noch von Verbandsvertretern und vor allem von Ideologen der Kapitalseite, zur Disposition gestellt. Jedenfalls gewann der amerikanische Pfad des „Niedriglohn-, Niedrigqualifikations-Preiswettbewerbs" an Attraktion und erschien manchen als ein durchaus nachahmenswertes Vorbild. Die Diskussion über eine verbesserte Wettbewerbsfähigkeit durch einen entsprechenden Pfadwechsel wurde offen geführt. Obwohl frühzeitig auf Schwierigkeiten und Kollateralschäden eines solchen Versuchs hingewiesen wurde, weil damit insbesondere das gerade in den Exportbranchen erfolgreich praktizierte High-Tech-Innovationsmodell gefährdet erschien, konnten wir damals auch diesen radikalen Umstieg beim Blick nach vorn nicht ausschließen.

Wenn ich in meiner Situationseinschätzung zur Jahrhundertwende von „Neue Unübersichtlichkeit" sprach (Schumann 2000, S. 112), so war dies den Ungleichzeitigkeiten, Widersprüchen und Unklarheiten geschuldet, mit denen in den Unternehmen auf die veränderten Rahmenbedingungen reagiert wurde. Beschäftigungspolitisch zeichneten sich bereits recht deutlich reine Verschlechterungen ab; leistungspolitisch wirkten nicht nur die traditionellen Intensivierungskonzepte weiter, sondern sie gewannen durch die Rekonventionalisierung der Arbeitspolitik und durch die Vereinnahmung der innovativen Arbeitsgestaltung noch sichtbar an Boden.
Zeitgleich zeigten sich aber auch bereits Grenzen der leistungspolitischen Instrumentalisierung der neuen Produktionskonzepte. Die Arbeitspolitik entwickelte viele Gesichter. Zu beobachten war gleichzeitig die weitere Durchsetzung der neuen Produktionskonzepte, der Japan-orientierte Lean Production Organisationsumbau, der arbeitsorganisatorisch vor allem die standardisierte Gruppenarbeit brachte und eben auch Retaylorisierung.

Der Blick auf betriebliche Rationalisierung zeigte also eine Pluralität von Gestaltungskonzepten, unterschiedliche Umsetzungsformen und heterogene Arbeitsfolgen. Ein vereinheitlichender Stilwandel, insbesondere der Arbeitspolitik, war nicht zu erkennen. Alle Bemühungen der Beraterszene, die in den Unternehmen gerade für Restrukturierungen immer mehr zu den „eigentlichen “ Experten avancierte, einen neuen „One best Way“ für Arbeitsgestaltung zu propagieren, waren nicht von Erfolg gekrönt. Je nach Einsatzbereich wurde vielmehr im Trial and Error vielfältig experimentiert.

Für den Kampf um Rationalisierung hieß das, dass sich Ende des Jahrhunderts die Fronten und Bündnispartner erneut verschoben hatten. Die gerade mit den neuen Produktionskonzepten erfolgreich praktizierte kooperativ-pragmatische Interessenvertretung, die auf gemeinsam erarbeitete und getragene arbeitspolitische Konzepte und tragfähige Kompromisslösungen setzte, erwies sich in der neuen Situation als defizitär. Jedenfalls pochte ein Teil der Vertreter der Kapitalseite auf seinen Machtzugewinn. Ihnen erschien das Konsens-Konzept zunehmend mehr als Hemmschuh für radikalere Erneuerungen und als Hindernis für einen angestrebten Pfadwechsel. Doch diese Positionen wurden nicht mehrheitsfähig. Die Vorteile des deutschen Produktionsmodells und die ja weiter höchst erfolgreich beschrittene High-Road-Strategie behielten die Oberhand.

Aber auch für die Gewerkschaftsseite wurden Mitwirkung und Mitverantwortung in den Unternehmen dort zum Problem, wo die Spielräume für vorzeigbare Erfolge und ein überzeugendes Win-WinArrangement sich verengten. Die Rolle vieler Betriebsräte bei innovativer Arbeitspolitik, die sie auch für die eigene Belegschaft zu Protagonisten dieser Rationalisierung gemacht hatte, wurde dann fragwürdig, wenn etwa bei Rücknahme von Höherqua- lifizierung, Partizipation und Selbstverantwortung entscheidende Eckpfeiler des Konzepts entfielen und durch leistungspolitische Instrumentalisierung die negativen Folgen für die Arbeit überwogen.

\section{Arbeit 2008 \\ 3.1 RAHMENBEDINGUNGEN: WACHSENDE DOMINANZ DES FINANZMARKT-KAPITALISMUS}

Dies ist also der historische Bezugsrahmen zur Beantwortung der Fragen: Zeigen sich gegenwärtig für die sozio-ökonomischen und politischen Rahmenbedingungen der Rationalisierung substanzielle, die betriebliche Rationalisierungspolitik bestimmende Veränderungen? Oder dokumentiert die erneute Aufnahme des Begriffs der „Neuen Unübersichtlichkeit“ eher Konstanz der Problemlagen?

Meine Einschätzung geht davon aus, dass sich einige der in den 1990er Jahren erkennbaren Tendenzen recht linear weiter durchgesetzt haben und gerade in ihren Arbeits- und Beschäftigungswirkungen zunehmend mehr Eindeutigkeit gewinnen. Die Marktökonomie hat in der Tat über die Produktionsökonomie „triumphiert“ (Dörre 2001; Sauer 2007), ohne dass damit freilich eine Determinierung des Wertschöpfungsprozesses erreicht wurde (Kädtler 2006). Immer deutlicher wird aber: „Am Beginn des 21. Jahrhunderts beginnt ein neues globales Akkumulationsregime zu tragen, unter dessen Bedingungen die erworbenen Statusrechte und eingelebten Ansprüche der Nutznießer des demokratischen Kapitalismus immer unrealistischer werden" (Streeck 2005, S. 36).

Politisch legitimatorisch ist dem Kapital das Spiel dadurch erleichtert, dass das Denken in Systemalternativen mit Verweis auf die Negativerfahrungen in der Geschichte weitgehend stillgesetzt wurde. Die Zukunft der Gesellschaft sehen zunehmend mehr Menschen eher skeptisch - utopische Energien scheinen aufgezehrt. Was immer sich dieser Kapitalismus leistet - Vorstellungen von einer besseren Welt haben sich zumindest als machbares politisches Projekt aus dem Alltag verflüchtigt (Assheuer 2008).

Gesellschaftliche Machtansprüche werden unverhohlener angemeldet. Soziale Gleichgültigkeit, ja sogar Kälte, kann gelebt 
werden. Immer krassere Disparitäten und Miseren in der Gesellschaft provozieren zwar zunehmend schärfere Kritik (siehe etwa die aktuellen Diskurse über Managergehälter, Armut und Ungerechtigkeit), doch deren politische Relevanz ist nicht absehbar. Die Feststellung von Gesine Schwan, dass „der Grundkonsens in dieser Gesellschaft ... für ein Zusammenleben in Freiheit, Gerechtigkeit und Solidarität ... auf dramatische Weise bis in die Gesellschaftsmitte erodiert (ist)“, und ihre Warnung: „Es gibt ein Maß von Ungerechtigkeit, an dem alle Gesellschaften zerbrechen" (Schwan 2008, S. 4), sind ernst zu nehmen. Das neoliberale Projekt jedenfalls, demzufolge Deregulierung und Marktfreiheit weltweite Fortschritte erschließen würden, hat seine Glaubwürdigkeit längst eingebüßt und gilt nur noch wenigen als aussichtsreiche Zukunftsvision.

Die einseitige Verteilung des gesellschaftlichen Reichtums, die den Beschäftigten Reallohnstagnation und Einkommensverluste in expandierenden Niedriglohnsektoren brachte und $\mathrm{zu}$ einem enormen Wachstum der Vermögenseinkommen führte, stärkt den Finanzmarkt. Unter dem „Regime des Vermögensbesitzes“ (Aglietta) wird der Kostendruck in den Unternehmen radikalisiert. Marktökonomie heißt dann: In den Unternehmenskalkulationen wird der Preis, der für ein Produkt im Markt durchsetzbar scheint, zur fixen Orientierungsgröße. Preis und angestrebte Gewinnspanne ergeben die Zielmarken, die nicht unterschritten werden dürfen. Die fortgeschrittenen Informations- und Transporttechnologien ermöglichen es, Produktionsprozesse auch über weiteste Entfernungen hinweg zu organisieren und dabei Güter und Arbeitskraft nahezu beliebig hin und her zu schieben. Beschäftigungs- und Leistungsbedingungen werden bei diesen Operationen zu den abhängigen Variablen. Denn wenn Finanzvorgaben nicht erreicht werden können, stehen Beschäftigungs-, Leistungs- und Entgeltstandards zur Disposition (Kädtler 2006).

Die Corporate Governance der Unternehmen hat sich verändert. Der Einfluss der Aufsichtsräte auf die Unternehmensführung wächst. Die Shareholder bringen sich direkter und intensiver in die Strategiebildung der Unternehmen und in deren Umsetzung ein und versuchen, ihre Rentabilitätsinteressen ohne Abstriche durchzusetzen. „Klassische Arbeitnehmerinteressen verlieren im Aufsichtsrat an Bedeutung" (Jürgens et al. 2007, S. 46).
In vielen Unternehmen haben sich zudem die Finanzvorstände, die ehemals „obersten Buchhalter“, zu Zentren der Macht gewandelt, die die Zielvorgaben in Aufnahme der Shareholder-Ansprüche und der Markterwartungen festlegen. Für die Produktionsverantwortlichen stehen die daraus abgeleiteten Produktionsvorgaben oft auch dann nicht zur Disposition, wenn ihre Einlösung mit immer größer werdenden Schwierigkeiten verbunden ist.

Mit den Machtverschiebungen in den Vorständen ist auch ein Funktionswandel verbunden. Wie eine Zielvorgabe in Planung und Fertigung tatsächlich eingelöst werden kann, wird immer stärker nach außen und unten verlagert. Dies ist die Stunde der externen Berater, die mit geliehener (Vorstands-)Autorität Restrukturierungsvorschläge einbringen, mit denen dann die Normhochsetzung bewältigt werden soll. Diese Funktionsverschiebungen lagern die Erarbeitung und Umsetzung der Problemlösungen bei den internen „Machern" ab. Das mittlere und untere Betriebsmanagement, die Planer, Entwickler, Systemregulierer, Maschinenbediener und Montagearbeiter sind mit aller auf diesen Ebenen verfügbaren Organisations- und Produzentenintelligenz gefordert, den erhöhten Ansprüchen gerecht zu werden. Man könnte auch sagen: den Letzten beißen die Hunde (Wagner 2007).

Insofern bringt dieser Funktionswandel gleichzeitig Kompetenzverschiebungen. Nicht nur die Produktionsvorstände, sondern auch die Akteure auf den gehobenen operativen Ebenen, etwa Werksleitungen, fungieren immer stärker als reine Promotoren der Vorgaben, die von der Marktund Renditelogik bestimmt werden. Deren Realisierung im Wertschöpfungsprozess ist an andere delegiert. Erst auf der Betriebsebene geht es dann um Antworten auf die Sperrigkeiten der eingesetzten Materialien, Techniken und auch Menschen. Entsprechend haben sich die Linienauseinandersetzungen zwischen arbeitspolitischen "Modernisierern" und "Traditionalisten“" eher auf diese unteren Ebenen verschoben. Damit kommen auch für die Betriebsräte andere Kooperationspartner ins Spiel, wenn es um die Erarbeitung rationalisierungspolitischer Lösungen geht.

Was heißen nun diese Unternehmensbedingungen für die Ratioanlisierungspolitik in den Arenen Beschäftigung, Leistung und Arbeit?

\subsection{BESCHÄFTIGUNGS- UND LEISTUNGSPOLITIK: LINEARE VERSCHIEBUNGEN - NEUE ÜBERSICHTLICHKEIT}

Beschäftigungspolitisch bleibt das Problem der Massenarbeitslosigkeit von höchster Priorität - trotz einer rückläufigen Arbeitslosenquote, die der weltweiten Konjunktur geschuldet ist. Immer mehr zeigen sich strukturell bedingte Verwerfungen besonders bei den Langzeitarbeitslosen. Und auch die Entwicklung der Beschäftigungsformen verdeutlicht, wie umfassend sich das Flexibilitätsprimat des Finanzkapitals durchzusetzen vermag: Steigende Zahlen für befristete Arbeitsverträge, Leiharbeit sowie Minijobs stehen für diese Tendenz ebenso wie der Abbau vertraglicher Sicherheiten und damit die Reduktion von Normalarbeitsverhältnissen (Dörre 2005).

Auch in der Leistungspolitik deutet die Richtung vieler Veränderungen recht unmissverständlich auf weitere Verschlechterungen hin. Historisch erkämpfte Standards für Arbeitszeiten und Leistungsvorgaben werden sukzessive zum Nachteil der Beschäftigten zurückgedrängt. Das meint konkret die Ausweitung von Schicht-, Nacht- und Sonntagsarbeit sowie Extensivierung der Arbeit durch erhöhte Wochenstundenzahlen. Beschäftigungsabbau kombiniert sich mit Arbeitsintensivierung. Wo es gelingt, die nicht formalisierbaren Leistungspotenziale der innovativen Arbeitspolitik leistungspolitisch zu instrumentalisieren, resultiert daraus ebenfalls Belastungserhöhung (dazu auch Seifert 2005).

Die neuen DGB-Umfragen belegen, dass die beschriebenen Verschlechterungserfahrungen zu großer mentaler Verunsicherung bei vielen Arbeitnehmern führen (DGB-Index 2008): Was wird die Zukunft bringen? Wie geht es weiter mit der Beschäftigung? Ist man auf Dauer den steigenden Anforderungen gewachsen? Wie diese latenten und manifesten Unzufriedenheiten in eine entsprechende Gewerkschafts- und Betriebspolitik umzusetzen sind, bleibt zu beantworten.

Wo der Kampf um Beschäftigung und Leistung heute geführt wird, werden die Grenzen einer pragmatisch-kooperativen, konsensorientierten Vertretungspolitik berührt. In vielen Fällen kann nur durch eine notfalls auch konfrontative Schutzpolitik eine Haltelinie gezogen und der Status 
quo gesichert werden. Bei allen zu berücksichtigenden historischen Differenzen: Die beschäftigungs- und leistungspolitisch verursachten Arbeitsverschlechterungen der letzten Jahre liefern beste Begründung für die Wiederaufnahme jener staatlichen Bemühungen der 1970er Jahre, mit denen durch gezielte HdA-Politik (Humanisierung der Arbeit) die krassesten Verwerfungen tayloristischer Arbeitsgestaltung konterkariert werden sollten (Matthöfer 1964). $\mathrm{Zu}$ erwarten ist freilich, dass sich die Unternehmen bei einer Mitwirkung heute noch sperriger anstellen werden als damals. Die Arbeitsmarktsituation sorgt dafür, dass sie der Schuh (bisher) allzu wenig drückt.

Im Kampffeld betrieblicher Rationalisierung, wenn es um Beschäftigung und Leistung geht, ergibt sich eine neue Übersichtlichkeit. Die Fronten sind zumeist klar gezogen, zumindest die Richtung der Forderungen ist eindeutig bestimmbar. Unklarheiten liegen darin, wie die Ansprüche bei gegebenen Kräfteverhältnissen durchzusetzen sind. Mobilisierung scheitert weniger an der Überzeugungskraft der Argumente und Forderungen als an der übergeordneten Sorge um Folgewirkungen für die betriebliche Position und die Beschäftigung. Mit einer schwierigen Lage im Wettbewerb und tendenziell bedrohter Existenz des Unternehmens, gleich ob als Realität oder auch nur als Suggestion eingebracht, können weitreichende Opfer überzeugend legitimiert werden. Forderungen, die die Bestandssicherung zu gefährden scheinen, haben es dann schwer, tragfähige Unterstützung zu finden.

Dennoch kann die Interessenvertretung in dieser veränderten Situation auf entsprechende politische Antworten nicht verzichten. Es geht um eine möglichst wirkungsvolle Abwehr- und Schutzpolitik. Das erwarten die Beschäftigten. Auch wenn dieser beschäftigungs- und leistungspolitische Ansatz zunächst defensiv ist, so kann er doch auch Bedeutung haben für die Arbeitspolitik. Soweit eine Unternehmenspolitik die Wettbewerbsfähigkeit durch Pfadwechsel zu einer Low-Cost-Produktionsstrategie anpeilt, stabilisiert Widerstand die Einhaltung des bisher betriebenen HighRoad-Weges. Eine zunächst auf Abwehr und Schutz gerichtete Politik schafft damit zugleich Grundlagen und Ansatzpunkte für innovative Arbeitspolitik.

\subsection{ARBEITSPOLITIK: KONZEPTPLURALITÄT, DIFFERENTE UMSETZUNGEN, WIDERSPRÜCH- LICHE WIRKUNGEN}

Für die heute in den Unternehmen praktizierte Arbeitspolitik ist Offenheit und Vielfalt kennzeichnend. Sie ergeben sich vor allem aus dem strukturellen Widerspruch, dass unter den Vorzeichen kurzfristiger Renditeoptimierung sich jedes Investment in den Einsatz und die Entwicklung von Humanressourcen als Kostenfaktor darstellt, den es möglichst klein zu halten und weiter zu reduzieren gilt. Alle arbeitspolitischen Konzepte demgegenüber, die ihre Voraussetzung im Erhalt und sogar Ausbau (sprich: zusätzliche Investitionen) in Humanressourcen haben, stehen entsprechend quer zu simplen Cost-Cutting-Strategien. Die durch die arbeitspolitische Gestaltung zu sichernde Leistungsfähigkeit des Wertschöpfungsprozesses limitiert also prinzipiell die Durchsetzung der Shareholder-Value-Ansprüche.

Eine generalisierte Low-Skill-Strategie erscheint nur denkbar unter Aufgabe des besonderen Profils der deutschen Industrieproduktion, das bisher deren weltweiten Erfolg verbürgt: Höchstansprüche an High-Tech-Produkte, hohe Innovationspotenziale für Produkte und Prozesse, qualitätssichernde, hochflexible Wertschöpfungsprozesse. Das sind die Anforderungen nicht nur im Maschinenbau, sondern genauso im Automobilbau und in anderen Branchen: Ohne ein hochqualifiziertes und einsatzbereites Arbeitskräftereservoir sind die Unternehmen kaum in der Lage, sich am Markt zu behaupten. Deswegen gilt nach wie vor die High-Road trotz aller Pfadwechseldebatten als die politikprägende Strategie.

Damit ist nicht außer Kraft gesetzt, dass überall dort, wo Low-Cost-Strategien möglich erscheinen, diese auch betrieben werden. Beschäftigungspolitisches Outsourcing steht dafür genauso wie rigide Taylorisierung mit entsprechender leistungspolitischer Nutzung. Doch für die Kernprozesse der Wertschöpfung in Deutschland geht es um eine Arbeitspolitik, die eine möglichst breite, das Know-how ausschöpfende Nutzung der Arbeitskraft sichert.

Schauen wir uns die heute in der deutschen Industrie praktizierten arbeitspolitischen Konzepte an, so variieren sie entsprechend dieser Vorgaben in beachtlicher Weise.

\section{ARBEITSPOLITISCHES SPEKTRUM}

Die Re-Taylorisierung als radikale Variante von Low-Cost-Strategien findet statt, hat aber keine Dominanz. Lean Production ist bestimmend geworden für die betriebliche Organisationsgestaltung, aber als arbeitspolitisches Konzept sind unter diesem Topos durchaus unterschiedliche Realisierungsformen zu beobachten. Auch die breit eingeführte Gruppenarbeit verbleibt im Variantenspektrum zwischen einerseits strukturkonservativen Konzepten, die auf strenge Arbeitsteilung, hohe Kontrolle und geringe Partizipation setzen, und strukturinnovativen Ansätzen, die Produktivität zu gewinnen suchen durch Funktions- und entsprechende Qualifikationserweiterungen, Hierarchieabbau und hohe Anteile an Selbstorganisation. Noch immer gibt es in den einzelnen Unternehmen Auseinandersetzungen um mehr oder weniger Gruppenautonomie - obwohl eine Tendenz in Richtung erweiterter Kontrollstrukturen und Rücknahme von Selbstverantwortung erkennbar ist.

\section{DER TOYOTA-WEG}

Das insbesondere in der Automobilindustrie propagierte Benchmark "Toyota-Weg“ heißt selbst in dieser Branche keineswegs einheitliche Arbeitsgestaltung. Eine stärkere Einbindung breiter Beschäftigtengruppen in Prozessoptimierungsaktivitäten oder ein neues betriebliches Selbstverständnis bezogen auf aktive Mitarbeit an Optimierungsbemühungen gelingen nur in Ausnahmefällen. Die für Toyota charakteristische Kombination aus materiellen Belohnungen (Rewards) und betrieblicher Vereinnahmung über kulturelle Traditionen, die auf eine Harmonisierung unterschiedlicher Interessen in einer Leistungsgemeinschaft setzt, erweist sich im deutschen Kontext als kaum praktikabel - und angesichts etablierter sozio-kultureller Standards letztlich auch nicht akzeptabel. Gerade in Deutschland darauf setzen zu wollen, heißt ein Spiel gegen die Gewerkschaften und das typische Arbeitnehmerverständnis zu eröffnen. Der Ideologieverdacht solcher Unternehmenskultur bewahrheitet sich allzu offenkundig (Peter 2007; Schumann et al. 2008).

\section{AUTO 5000}

Für den Automobilbau kann das VW-Projekt Auto 5000, in dem hier interessieren- 
den Zeitraum in Wolfsburg in enger $\mathrm{Zu}$ sammenarbeit zwischen Unternehmensleitung, Betriebsrat und IG Metall geplant und realisiert, in Bezug auf Arbeits- und Betriebsgestaltung als das avancierteste Modell einer innovativen Arbeitspolitik gelten (Schumann et al. 2006). Das Vorhaben war sowohl in seinen ökonomischen wie auch in seinen Humanisierungsansprüchen insgesamt sehr erfolgreich. Es repräsentiert eine Good Practise, die entlang der neuen Produktionskonzepte gedacht wurde, betriebsorganisatorisch aber deutliche Erweiterungen aufnahm. Dies gilt insbesondere für die auf drei Stufen reduzierte Werkshierarchie, die weitgehende $\mathrm{Zu}$ sammenführung von indirekten und direkten Arbeitsprozessen unter der Regie der Fertigung sowie status- und funktionsgruppenübergreifende horizontale Kooperationsstrukturen. Die Zugewinne an Autonomie für selbstständiges Verhalten werden in diesem Projekt nicht durch leistungspolitische Vereinnahmung wieder entwertet. Im VW-Projekt Auto 5000 wurden zwar Toyota-Erfahrungen systematisch berücksichtigt und viele Toyota-Tools genutzt, bei der Unternehmenskultur werden jedoch deutlich andere Akzente gesetzt. Hier wird eine "mitbestimmungsjustierte“ Einbeziehung der Beschäftigten praktiziert. Innerbetriebliche Konsensbildung und Leistungsbereitschaft werden nicht über ideelle „Vergemeinschaftung “ erreicht, sondern durch reale Arbeitsverbesserungen bei Offenlegung der gemeinsamen und auch unterschiedlichen Interessen des Unternehmens und der Beschäftigten sowie durch mitbestimmungsbasiertes rationales Aushandeln und offene Kompromissfindung, was Problemlösungen nach ausgetragenen Konflikten einschließt. Eine stärkere Einbindung von Beschäftigten in Prozessoptimierungsaktivitäten wird hierbei nicht zuletzt durch die etablierten innovativen Arbeitsformen und Organisationslösungen erreicht.

Bisher bleibt das Projekt zwar ohne Nachfolge, doch es enthält hohe Lernanreize für künftige arbeitspolitische Konzeptionierungen, und es ist appellationsfähig. Denn es liefert überzeugende Begründungen für den Ausbau innovativer Arbeitspolitik mit konkurrenzfähiger, ja konkurrenzüberlegener Wirkung.

Heute ist in den Unternehmen, weniger als vor zehn Jahren, die Arbeitspolitik nicht durch konzeptionell ausdifferenzierte Linienauseinandersetzungen bestimmt, son- dern durch eine Mischung aus pragmatischem Weitermachen und Detail-Korrekturen. Ein neuer Königsweg, den zunächst in den 1980er Jahren die neuen Produktionskonzepte zu bieten schienen und für viele dann in den 1990er Jahren Lean Production, ist weder verfügbar noch in Sicht. Auch im Selbstverständnis vieler Manager dominiert „Unübersichtlichkeit“, wie tragfähige arbeitspolitische Lösungen in $\mathrm{Zu}-$ kunft aussehen könnten.

\section{SUBJEKTIVIERUNG}

Dies zeigt sich besonders deutlich im Umgang mit dem Subjektivierungsproblem. Immer noch changiert die arbeitspolitische Gestaltung zwischen Konzepten, die auf die erweiterte Wertschätzung der menschlichen Arbeit setzen und dafür die Objektrolle einschränken und solchen, die mit ihrem leistungspolitisch rigorosen Zugriff arbeitspolitisch eingeräumte Autonomiespielräume wieder zurücknehmen. Deutlich ist zu erkennen, dass der Instrumentalisierung der Subjektivierung Grenzen gesetzt sind. In vielen Produktionsprozessen ist eine Optimierung oder auch nur Aufrechterhaltung der Planungs- und Fertigungsprozesse ohne reale, leistungspolitisch nicht wieder kanalisierte Freiräume bei der Arbeitsausführung und selbstständige Problemlösung nicht zu gewährleisten. Die Entwickler, Planer oder auch Systemregulierer der automatisierten Fertigung können am Gängelband formalisierter Vorgaben, hierarchischer Kontrollen und zeitökonomischer Einengungen oft nicht einmal suboptimal agieren.

Im Umgang mit der Subjektivierung müssen die Unternehmen noch eine zusätzliche Aufgabe lösen. Im TaylorismusFordismus wurde das sogenannte Transformationsproblem (neben dem Kauf der Arbeitskraft ist eine anforderungsgemäße reale Entäußerung ihrer Fähigkeiten erst durch gezielte betriebliche Angebote zu gewährleisten) durch die im Arbeitsvertrag vereinbarten materiellen Gratifikationen und Regelungen, d.h. durch Zugeständnisse bei Lohn und Arbeitsbedingungen, gelöst. Das hat lange Zeit ausgereicht, weil es wenigstens durch Sicherheiten Anerkennung gewährleistete.

Entsprechend der arbeitsinhaltlichen Aufwertungsprämissen bei innovativer Arbeitspolitik wird mit diesem Ansatz das Transformationsproblem auf neuem Niveau angegangen: Arbeitsbereitschaft und
Arbeitsengagement werden nicht mehr nur durch materielle Rewards, sondern auch durch arbeitsinhaltliche Bedingungen gewährleistet (Minssen 2006). Der Wunsch nach Anerkennung durch die Arbeit wird damit breiter eingelöst. Wenn der finanzmarktgesteuerte Kapitalismus zunehmend mehr die materiellen Bedingungen bei Lohn, Beschäftigung und Leistung verschlechtert und gleichzeitig die SubjektAnsprüche durch re-taylorisierte Arbeitsgestaltung oder durch leistungspolitische Instrumentalisierung zurücknimmt, so könnte dies die Transformation von Arbeitskraft in Arbeitsverausgabung empfindlich stören. Auch dieser Sachverhalt stützt arbeitspolitische Konzepte, die die Subjektansprüche der Beschäftigten positiv einlösen.

\subsection{ZWISCHENFAZIT}

Das letzte Jahrzehnt hat gezeigt, dass die unterschiedlichen Erwartungen an und Bedingungen von Arbeitspolitik die bereits in den 1990er Jahren zu beobachtende Konzeptvielfalt kaum verändern. Eine stilbildende Arbeitspolitik hat sich dabei nicht herausgebildet. Insofern macht es auch keinen Sinn, von einer neuen arbeitspolitischen Inkubationszeit zu sprechen. Dafür fehlt das Orientierung bietende Leitprojekt. Ein Pfadwechsel steht gegenwärtig ernsthaft nicht zur Debatte. Ausprobieren ist angesagt, vielerorts kann man auch durchaus sagen: Durchwurschteln. Für die Auflösung des Widerspruchs zwischen Kosten- oder Humanressourcen-Primat gibt es keinen Algorithmus. Privilegiert man einseitig die Einsparungsseite, so werden die gewünschten Resultate des Wertschöpfungsprozesses gefährdet und man landet zwangsläufig wieder beim Taylorismus mit allen erkannten Defiziten.

Anders als bei der Beschäftigungs- und Leistungspolitik ist bei der Arbeitspolitik die Frontstellung zwischen Unternehmen und Belegschaft offener, und die Forderungen müssen sich nicht widersprüchlich begegnen. Das Management kann keinen One-best-Way offerieren, der zumindest aus der eigenen Interessenperspektive optimal wäre. Die Unternehmen suchen für ihre jeweiligen Fertigungsbedingungen und Produktspektren arbeitspolitisch tragfähige Lösungen. Dies gewährleistet weiterhin Win-Win-Konstellationen und entsprechend ausgehandelte Rationalisierungskompromisse. Das von der Interessenver- 
tretung einzubringende Konzept einer innovativen Arbeitspolitik kann zu Recht darauf verweisen, dass mit ihm gleichermaßen Wirtschaftlichkeit und Sozialverträglichkeit verbessert werden. Die Wettbewerbsvorteile einer auf Produzentenintelligenz und Selbstständigkeit aufbauenden Arbeitspolitik sind durch viele, auch betriebswirtschaftlich evaluierte GoodPractice-Beispiele nicht mehr nur Anspruch, sondern nachgewiesen (Kuhlmann et al. 2004).

Andererseits ist aus der Begleitforschung ebenfalls bekannt, dass die Betriebsräte bei angemessen konzipierter, konsequent umgesetzter und leistungspolitisch nicht instrumentalisierter innovativer Arbeitspolitik auf die aktive Unterstützung dieser Politik durch die Beschäftigten rechnen können. Diese erkennen dann durchaus mehrheitlich die damit auch für sie erreichbaren Vorteile.

Die Durchsetzung des Konzepts einer innovativen Arbeitspolitik könnte in den Unternehmen wirkungsvoll durch die Arbeitnehmervertreter im Aufsichtsrat unterstützt werden. Der erweiterte Einfluss der Aufsichtsräte auf die Unternehmensführung, von Shareholdern genutzt, um ihren Renditeinteressen Geltung zu verschaffen, gibt in mitbestimmten Unternehmen der Arbeitnehmerbank durchaus die Chance, mit Nachdruck ihre arbeitspolitischen Strategievorschläge einzubringen. Diese sind umso durchsetzungsfähiger, wenn sie im Konzept der innovativen Arbeitspolitik nicht nur ihre unmittelbaren Klientelinteressen bedienen, sondern auch für das Unternehmen eine Erfolgsabsicherung bei Produktivität, Qualität, Innovationsfähigkeit, Flexibilität und Ressourcenschonung anzubieten haben (Schumann 2008).

Die Gewerkschaften können bei der Arbeitspolitik davon ausgehen, dass die immer anspruchsvoller werdenden Wertschöpfungsprozesse in Deutschland nur in guter Kooperation mit High-Skill-Belegschaften zu bewältigen sind. Die gewerkschaftliche Strategie zur Re-Vitalisierung der Arbeitspolitik kann auf dieser Erfahrung aufbauen. Der durchaus als gescheitert geltende Pfadwechsel dokumentiert auch die Stärke der qualitativen Potenziale im Wertschöpfungsprozess und stützt eine Politik, die die Produktivkraft der lebendigen Arbeit durch Human-ResourceDevelopment zur Entfaltung zu bringen sucht.

Für die Gewerkschaften ermöglicht dies eine Doppelstrategie (Kuhlmann 2007). Neben der eher defensiven leistungs- und beschäftigungspolitischen Schutz-Politik ist gleichzeitig eine offensive Arbeitspolitik in die Unternehmen einzubringen. Das meint für Arbeitspolitik ein Festhalten an einem konsensorientierten, kooperativen Politikansatz - soweit jedenfalls im gemeinsamen arbeitspolitischen Konzept an den Verbesserungen für die Arbeitsseite festgehalten werden kann. Dabei gilt: Mit der Rücknahme des Doppelanspruchs von Wirtschaftlichkeit und Sozialverträglichkeit durch innovative Arbeitspolitik ginge auch ihre ökonomische Leistungsfähigkeit im Wertschöpfungsprozess verloren.

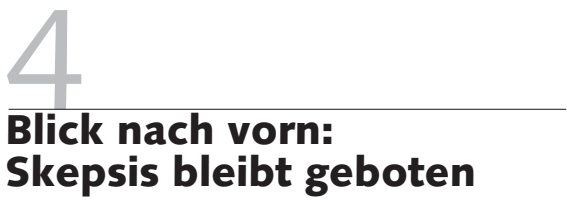

Die Frage nach der Deutungshoheit war gestellt. Sie einzulösen, bedeutet im Kontext einer Re-Vitalisierung der Arbeitspolitik an einer Definition von Arbeit festzuhalten, die sich den ökonomischen Anforderungen des Wertschöpfungsprozesses stellt, mit ihrer Einlösung aber gleichzeitig die gesellschaftliche Anerkennung in und durch Arbeit als legitime Forderung sichert. Der Kampf um die richtige Arbeitspolitik muss auch ein Kampf um mehr Arbeitswürde sein (Negt 2001). Diese Auseinandersetzung kann mit realistischen Zielen angetreten werden und mit breiter Unterstützung der Arbeitnehmerschaft und in der Gesellschaft rechnen.

Ginge es um gut begründbare, rationale Entscheidungen, spräche alles für ein tragfähiges Bündnis zwischen Kapital und Arbeit zur Weiterentwicklung der Konzepte innovativer Arbeitspolitik. Wir wissen aber aus den Rationalisierungserfahrungen der letzten 20 Jahre, dass schon mit den Neuen Produktionskonzepten kein arbeitspolitischer Selbstläufer entwickelt worden ist. Offenkundig überschätzt man damit die Lernfähigkeit des Kapitalismus und übersieht die System-Irrationalismen. Allein die Kurzfrist-Interessen des Shareholder-Value-Kalküls entschlüsseln diesen Sachverhalt nicht. Es drückt sich darin wohl auch das weiterhin bestehende Grundmisstrauen der Kapitalseite gegenüber einer Arbeitnehmerschaft aus, die im Zuge der Neuen Produktionskonzepte selbstständiger agieren konnte. Der in den letzten Jahren wieder gestärkte Kontrollanspruch, möglichst alles selbst im Griff haben zu wollen, entspringt vermutlich auch solchen Sichtweisen. Der Widerstand in der Biedenkopf-Mitbestimmungskommission gegen eine gemeinsame Lösung wurde durch derartige Haltungen bestimmt (Schumann 2007). Bleibt also die Frage, wie viel Einbußen an Produktivität und Innovationskraft sich Deutschland auf Dauer leisten kann. Eine erfolgreiche Kapitalverwertung am Standort Deutschland ist gerade unter den Bedingungen der weltweiten Konkurrenz auf die volle Nutzung der Produzentenintelligenz verwiesen. Für die Re-Vitalisierung der gewerkschaftlichen Arbeitspolitik liegt jedenfalls gerade in diesem Sachverhalt eine zu nutzende Chance. 
Assheuer, T. (2008): Der große Ausverkauf, in: Die Zeit vom 27.03.2008, S. 49

Baethge, M./Kern, H./Schumann, M. (1988): Arbeit und Gesellschaft, Rückblicke und Ausblicke aus 25 Jahren Göttinger Soziologischer Forschung, Soziologisches Forschungsinstitut Göttingen - Mitteilungen 15, Göttingen, S. 1-15

Detje, R./Pickshaus, K./Urban, H.-J. (Hrsg.) (2005): Arbeitspolitik kontrovers. Zwischen Abwehrkämpfen und Offensivstrategien, Hamburg DGB-Index Gute Arbeit (2008): Der Report. Wie die Beschäftigten die Arbeitswelt in Deutschland beurteilen, was sie sich von Guter Arbeit erwarten, Berlin

Dörre, K. (2001): Das Deutsche Produktionsmodell unter dem Druck des Shareholder Value, in: Kölner Zeitschrift für Soziologie und Sozialpsychologie 4, Köln, S. 675-704

Dörre, K. (2005): Prekarität - Eine arbeitspolitische Herausforderung, in: WSI-Mitteilungen 5, S. 250-258

Dörre, K./Röttger, B. (Hrsg.) (2003): Das neue Marktregime. Konturen eines nachfordistischen Produktionsmodells, Hamburg

Dröge, K./Marrs, K./Menz, W. (Hrsg.) (2008): Rückkehr der Leistungsfrage. Leistung in Arbeit, Unternehmen und Gesellschaft, Berlin Dunkel, W./Sauer, D. (Hrsg.) (2006): Von der Allgegenwart der verschwindenden Arbeit. Neue Herausforderungen für die Arbeitsforschung, Berlin

Fergen, A./Pickshaus, K. (2008): Der Arbeit wieder ein gesundes Maß geben - neue arbeitspolitische Ansätze für "Gute Arbeit" , in: Wagner, H. (Hrsg.): Arbeit und Leistung - gestern und heute. Ein gewerkschaftliches Politikfeld, Hamburg

Habermas, Jürgen (1985): Die Neue Unübersichtlichkeit, Frankfurt a. M. Huber, B. (2008): Thesenpapier (unveröffentlicht), Frankfurt a. $M$. Huber, B./Burkhard, O./Wagner, H. (Hrsg.) (2006): Perspektiven der Tarifpolitik. Im Spannungsfeld von Fläche und Betrieb, Hamburg Huber, B./Schwitzer, H. (2008): Vorwort, in: Wagner, H. (Hrsg.): Arbeit und Leistung - gestern und heute. Ein gewerkschaftliches Politikfeld, Hamburg

Jürgens, U./Lippert, I./Gaeth, F. (2007): Information, Kommunikation und Wissen im Mitbestimmungssystem, Berlin

Kädtler, J. (2006): Sozialpartnerschaft im Umbruch, Industrielle Beziehungen unter den Bedingungen von Globalisierung und Finanzmarktkapitalismus, Hamburg

Kleemann, F./Matuschek, I./Voß, G. G. (2002): Subjektivierung von Arbeit - Ein Überblick zum Stand der soziologischen Literatur, in: Moldaschl, M./Voß, G. G. (Hrsg.): Subjektivierung von Arbeit, München/Mering Kratzer, N./Menz, W./Nies, S./Sauer, D. (2008): Leistungspolitik als Feld "Umkämpfter Arbeit“, in: Prokla 150, S. 11-26 Kuhlmann, M. (2007): Jenseits von Gruppenarbeit - Perspektiven der Arbeitspolitik, in: Peter, G. C. (Hrsg.): Grenzkonkflikte der Arbeit. Die Herausbildung einer neuen europäischen Arbeitspolitik, Hamburg, S. 129-143

Kuhlmann, M./Sperling, H.-J./Balzert, H. (2004): Konzepte innovativer Arbeitspolitik, Good-Practice-Beispiele aus dem Maschinenbau, der Automobil-, Elektro- und Chemischen Industrie, Berlin
Kurz, C. (1999): Repetitivarbeit - unbewältigt. Betriebliche und gesellschaftliche Entwicklungsperspektiven eines beharrlichen Arbeitstyps, Berlin

Matthöfer, H. (1964): Für menschenwürdige Arbeitsbedingungen, in: Der Gewerkschaftler 7

Minssen, H. (2006): Crisis: What Crisis? - Zur Situation der Arbeits- und Industriesoziologie, in: Arbeit 4, S. 259-272

Negt, O. (2001): Arbeit und menschliche Würde, Göttingen

Peter, G. (Hrsg.) (2007): Grenzkonflikte der Arbeit. Die Herausbildung einer neuen europäischen Arbeitspolitik, Hamburg

Prokla-Redaktion (2008): Editorial, in: Prokla 150, Münster, S. 2-10 Sauer, D. (2007): Vermarktlichung und Politik - Arbeitspolitik unter den Bedingungen indirekter Steuerung, in: Peter, G. (Hrsg.): Grenzkonflikte der Arbeit, Hamburg

Schumann, M. (1998): Rücknahme der Entwarnung, in: Gewerkschaftliche Monatshefte 6-7, S. 457-460

Schumann, M. (2000): Industriearbeit zwischen Entfremdung und Entfaltung, Soziologisches Forschungsinstitut Göttingen - Mitteilungen 28,

S. $103-112$

Schumann, M. (2003): Metamorphosen von Industriearbeit und Arbeiterbewusstsein, Kritische Industriesoziologie zwischen Taylorismusanalyse und Mitgestaltung innovativer Arbeitspolitik, Hamburg

Schumann, M. (2008): Die Arbeitnehmerbank muss ihre Kernkompetenzen ausspielen, in: Mitbestimmung 1+2, S. 38-41

Schumann, M./Baethge-Kinsky, V./Kuhlmann, M./Kurz, C./Neumann, U. (1994): Trendreport Rationalisierung, Automobilindustrie, Werkzeugbau, Chemische Industrie, Berlin

Schumann, M./Kern, H. (1984): Das Ende der Arbeitsteilung? Rationalisierung in der industriellen Produktion, München

Schumann, M./Kuhlmann, M./Sanders, F./Sperling, H.-J. (Hrsg.) (2006): Auto 5000: ein neues Produktionskonzept. Die deutsche Antwort auf den Toyota-Weg?, Hamburg

Schumann, M. (2007): Warum Mitbestimmung?, in: Personalführung 3, S. 60-67

Schumann, M./Kuhlmann, M./Sperling, H.-J. (2008): Zwischen Toyota und Tradition - das VW-Projekt "Auto 5000" als mitbestimmungsjustierte Unternehmenskultur, in: Brinkmann, U. (Hrsg.): Unternehmenskultur und Mitbestimmung (im Erscheinen).

Schwan, G. (2008): In einer wölfischen Welt, in: Die Zeit vom 12.06.2008, S. 4

Seifert, H. (Hrsg.) (2005): Flexible Zeiten in der Arbeitswelt, Frankfurt a. M. Streeck, W. (2005): Vom kurzen Traum zum langen Alptraum. Die globale Landnahme des Kapitalismus verändert die Verhältnisse radikal, in: Mitbestimmung 9 , S. 32

Wagner, G. (2007): Ein „neuer Geist des Kapitalismus“?. Paradoxien der Selbstverantwortung, in: Österreichische Zeitschrift für Soziologie 3,

S. 3-21

Wagner, H. (Hrsg.) (2008): Arbeit und Leistung - gestern und heute. Ein gewerkschaftliches Politikfeld, Hamburg 